Appendix S1. Tribe Aedini generic, subgeneric and species names proposed herein. As appropriate, species endings are written to agree in gender with their respective genera and author parentheses are provided for species described in a genus different than in the current classification.

\title{
Genus Aedes
}

Subgenus Abraedes Zavortink, 1970

papago Zavortink, 1970

Subgenus Acartomyia Theobald, 1903

mariae (Sergent \& Sergent, 1903)

phoeniciae Coluzzi \& Sabatini, 1968

zammitii (Theobald, 1903)

Subgenus Aedes Meigen, 1818

alexandrei Gornostaeva, 2005

cinereus Meigen, 1818

dahuricus Danilov, 1987

dmitryi Gornostaeva, 2005

esoensis Yamada, 1921

geminus Peus, 1970

mubiensis Luh \& Shih, 1958

nataliae Gornostaeva, 2005

rossicus Dolbeskin, Gorickaja \& Mitrofanova, 1930

sasai Tanaka, Mizusawa \& Saugstad, 1975

valeryi Gornostaeva, 2005

yamadai Sasa, Kano \& Takahasi, 1950

Subgenus Aedimorphus Theobald, 1903

abnormalis (Theobald, 1909)

abnormalis kabwachensis Edwards, 1941

aerarius McIntosh, 1975

albodorsalis Fontenille \& Brunhes, 1985

alboscutellatus (Theobald, 1905)

alboventralis (Theobald, 1910)

argenteoscutellatus Carter \& Wijesundara, 1948

bambiotai Geoffroy, 1987

bancoi Geoffroy, 1987 
bevisi (Edwards, 1915)

caecus (Theobald, 1901)

caliginosus (Graham, 1910)

centropunctatus (Theobald, 1913)

culicinus Edwards, 1922

cumminsii (Theobald, 1903)

cumminsii mediopunctatus (Theobald, 1909)

dalzieli (Theobald, 1910)

davidi Basio, 1971

dentatus (Theobald, 1904)

domesticus (Theobald, 1901)

durbanensis (Theobald, 1903)

durbanensis angolae Ribeiro \& Ramos, 1974

ebogoensis Rickenbach \& Ferrara, 1965

eritreae Lewis, 1942

fowleri (de Charmoy, 1908)

gibbinsi Edwards, 1935

gouldi Reinert, 1972

grjebinei Hamon, Taufflieb \& Maillot, 1957

hirsutus (Theobald, 1901)

hirsutus adenensis Edwards, 1941

holocinctus Edwards, 1941

jamesi (Edwards, 1914)

karooensis Muspratt, 1961

leesoni Edwards, 1932

leptolabis Edwards, 1936

leucarthrius (Speiser, 1909)

longiseta Edwards, 1936

lowisii (Theobald, 1910)

mansouri Qutubuddin, 1959

masoalensis Fontenille \& Brunhes, 1985

mathioti Fontenille \& Brunhes, 1985

mattinglyi Hamon \& Rickenbach, 1954

mediolineatus (Theobald, 1901)

natronius Edwards, 1932

nigricephalus (Theobald, 1901)

nigrostriatus (Barraud, 1927)

oakleyi Stone, 1939

ochraceus (Theobald, 1901)

orbitae Edwards, 1922

ovazzai Hamon \& Adam, 1959

pachyurus Edwards, 1936

pallidostriatus (Theobald, 1907)

pampangensis (Ludlow, 1905) 


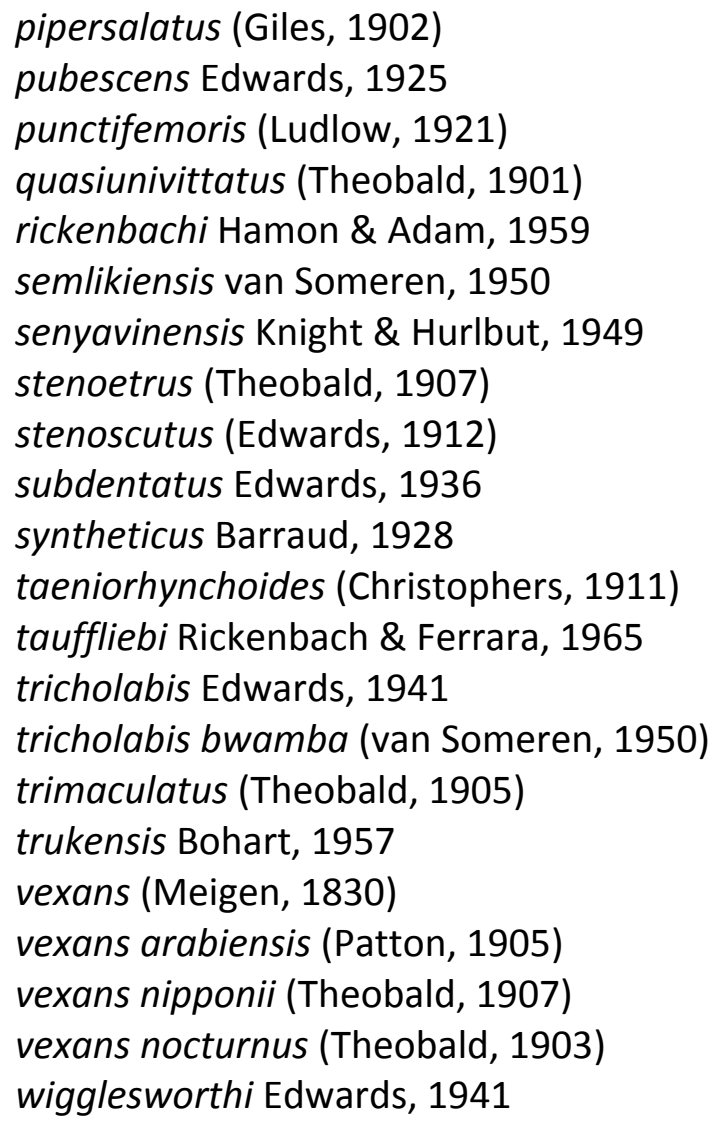

Subgenus Alanstonea Mattingly, 1960

brevitibia (Edwards, 1914)

treubi (de Meijere, 1910)

Subgenus Albuginosus Reinert, 1987

capensis Edwards, 1924

gilliesi van Someren, 1962

haworthi Edwards, 1923

kapretwae Edwards, 1941

kennethi Muspratt, 1956

marshallii (Theobald, 1901)

ngong van Someren, 1950

stokesi Evans, 1929

teesdalei van Someren, 1954

Subgenus Ayurakitia Thurman, 1954

griffithi (Thurman, 1954)

peytoni Reinert, 1972 
Subgenus Aztecaedes Zavortink, 1972

ramirezi Vargas \& Downs, 1950

Subgenus Belkinius Reinert, 1982

aurotaeniatus Edwards, 1922

Subgenus Bifidistylus Reinert, Harbach \& Kitching, 2009

boneti Gil Collado, 1936

boneti kumbae Chwatt, 1948

lamborni Edwards, 1923

Subgenus Borichinda Harbach \& Rattanarithikul, 2007

cavernicolus (Rattanarithikul \& Harbach, 2007)

Subgenus Bothaella Reinert, 1973

alongi Galliard \& Ngu, 1947

brownscutumus Dong, Zhu \& Dong, 1999

eldridgei Reinert, 1973

helenae Reinert, 1973

kleini Reinert, 1973

manhi (Harbach \& Cook, 2010)

Subgenus Bruceharrisonius Reinert, 2003

alektorovi Stackelberg, 1943

aureostriatus (Doleschall, 1857)

christophersi Edwards, 1922

doonii Wattal, Bhatia \& Kalra, 1958

greenii (Theobald, 1903)

hurlbuti Lien, 1967

okinawanus Bohart, 1946

taiwanus Lien, 1968

Subgenus Cancraedes Edwards, 1929

cancricomes Edwards, 1922

curtipes Edwards, 1915

indonesiae Mattingly, 1958 
kohkutensis Mattingly, 1958

mamoedjoensis Mattingly, 1958

masculinus Mattingly, 1958

palawanicus Mattingly, 1958

penghuensis Lien, 1968

simplex (Theobald, 1903)

thurmanae Mattingly, 1958

Subgenus Catageiomyia Theobald, 1903

adami Geoffroy, 1971

argenteopunctatus (Theobald, 1901)

bedfordi Edwards, 1936

chamboni Cornet, 1968

dialloi Hamon \& Brengues, 1965

falabreguesi Hamon, 1957

filicis Ingram \& de Meillon, 1927

grenieri Hamon, Service, Adam \& Taufflieb, 1961

hopkinsi Edwards, 1936

insolens Edwards, 1936

irritans (Theobald, 1901)

lokojoensis Service, 1959

lottei Hamon \& Brengues, 1965

microstictus Edwards, 1936

minutus (Theobald, 1901)

mixtus Edwards, 1936

mutilus Edwards, 1936

nyounae Hamon \& Adam, 1959

phyllolabis Edwards, 1929

pseudotarsalis van Someren, 1946

punctothoracis (Theobald, 1909)

reali Hamon \& Adam, 1959

smithburni van Someren, 1950

tarsalis (Newstead, 1907)

veeniae McIntosh, 1975

wendyae Service, 1959

yangambiensis de Meillon \& Lavoipierre, 1944

yvonneae Edwards, 1941

Subgenus Catatassomyia Dyar \& Shannon, 1925

meronephada (Dyar \& Shannon, 1925)

Subgenus Christophersiomyia Barraud, 1923 
annulirostris (Theobald, 1905)

chionodes Belkin, 1962

gombakensis Mattingly, 1959

ibis Barraud, 1931

thomsoni (Theobald, 1905)

Subgenus Coetzeemyia Huang, Mathis \& Wilkerson

fryeri (Theobald, 1912)

Subgenus Collessius Reinert, Harbach \& Kitching, 2006

banksi Edwards, 1922

elsiae (Barraud, 1923)

elsiae vicarius Lien, 1968

hatorii Yamada, 1921

macdougalli Edwards, 1922

macfarlanei (Edwards, 1914)

ningheensis Lei, 1989

pseudotaeniatus (Giles, 1901)

shortti (Barraud, 1923)

tonkinensis Galliard \& Ngu, 1947

Subgenus Cornetius Huang, 2005

cozi Cornet, 1973

Subgenus Dahliana Reinert, Harbach \& Kitching, 2006

echinus (Edwards, 1920)

geniculatus (Olivier, 1791)

gilcolladoi (Sánchez-Covisa Villa, Rodríguez Rodríguez \& Guillén Llera, 1985)

Subgenus Danielsia Theobald, 1904

albotaeniatus (Leicester, 1904)

harperi Knight, 1948

lepchanus (Barraud, 1923)

Subgenus Dendroskusea Edwards, 1929

kanarensis Edwards, 1934

micropterus (Giles, 1901) 
periskelatus (Giles, 1902)

ramachandrai Reuben, 1967

reginae Edwards, 1922

Subgenus Diceromyia Theobald, 1911

adersi (Edwards, 1917)

bananea Wolfs, 1958

cordellieri Huang, 1986

coulangesi Rodhain \& Boutonnier, 1983

fascipalpis (Edwards, 1912)

flavicollis Edwards, 1928

furcifer (Edwards, 1913)

grassei Doucet, 1951

madagascarensis van Someren, 1949

mefouensis Ferrara, 1974

sylvaticus Brunhes, 1983

taylori Edwards, 1936

tiptoni Grjebine, 1953

zethus de Meillon \& Lavoipierre, 1944

Subgenus Dobrotworskyius Reinert, Harbach \& Kitching, 2006

alboannulatus (Macquart, 1850)

milsoni (Taylor, 1915)

occidentalis (Skuse, 1889)

rubrithorax (Macquart, 1850)

rupestris Dobrotworsky, 1959

subbasalis Dobrotworsky, 1962

tubbutiensis Dobrotworsky, 1959

Subgenus Downsiomyia Vargas, 1950

albolateralis (Theobald, 1908)

alboniveus Barraud, 1934

axitiosus Kulasekera, Knight \& Harbach, 1990

dorseyi Knight, 1946

ganapathi Colless, 1958

harinasutai Knight, 1978

idjenensis Brug, 1934

inermis Colless, 1958

lacteus Knight, 1946

laoagensis Knight, 1946

leonis Colless, 1958 
litoreus Colless, 1958

mikrokopion Knight \& Harrison, 1988

mjobergi (Edwards, 1926)

mohani Knight, 1969

nipponicus LaCasse \& Yamaguti, 1948

nippononiveus Sasa \& Nakahashi, 1952

nishikawai Tanaka, Mizusawa \& Saugstad, 1979

niveoides Barraud, 1934

niveus (Ludlow, 1903)

novoniveus Barraud, 1934

omorii Lien, 1968

pexus Colless, 1958

pseudoniveus (Theobald, 1905)

saperoi Knight, 1946

shehzadae Qutubuddin, 1972

sinensis Chow, 1950

subniveus Edwards, 1922

vanus Colless, 1958

watteni Lien, 1968

Subgenus Edwardsaedes Belkin, 1962

bekkui Mogi, 1977

imprimens (Walker, 1861)

pingpaensis Chang, 1965

Subgenus Elpeytonius Reinert, Harbach \& Kitching, 2009

apicoannulatus (Edwards, 1912)

simulans (Newstead \& Carter, 1911)

Subgenus Finlaya Theobald, 1903

alocasicola Marks, 1947

ananae Knight \& Laffoon, 1946

avistylus Brug, 1939

bougainvillensis Marks, 1947

burnetti Belkin, 1962

croceus Knight \& Laffoon, 1946

dobrotworskyi Marks, 1958

fijiensis Marks, 1947

flavipennis Giles, 1904

franclemonti Belkin, 1962

freycinetiae Laird, 1957 
fuscipalpis Belkin, 1962

fuscitarsis Belkin, 1962

gahnicola Marks, 1947

gani Bonne-Wepster, 1940

gressitti Bohart, 1957

hollingsheadi Belkin, 1962

horotoi Taylor, 1972

hui Bohart, 1957

josephinae Marks, 1958

knighti Stone \& Bohart, 1944

kochi (Dönitz, 1901)

lewelleni Starkey \& Webb, 1946

luteus (Ludlow, 1905)

maffii Taylor \& Tenorio, 1974

medleri Knight \& Laffoon, 1946

neogeorgianus Belkin, 1962

oceanicus Belkin, 1962

poicilius (Theobald, 1903)

samoanus (Grünberg, 1913)

schlosseri Belkin, 1962

solomonis Stone \& Bohart, 1944

sorsogonensis Bañez \& Jueco, 1966

stonei Knight \& Laffoon, 1946

tutuilae Ramalingam \& Belkin, 1965

wallacei Edwards, 1926

Subgenus Fredwardsius Reinert, 2000

vittatus (Bigot, 1861)

Subgenus Georgecraigius Reinert, Harbach \& Kitching, 2006

atropalpus (Coquillett, 1902)

epactius Dyar \& Knab, 1908

fluviatilis (Lutz, 1904)

Subgenus Geoskusea Edwards, 1929

baisasi Knight \& Hull, 1951

becki Belkin, 1962

daggyi Stone \& Bohart, 1944

fimbripes Edwards, 1924

kabaenensis Brug, 1939

longiforceps Edwards, 1929 
lunulatus King \& Hoogstraal, 1946

perryi Belkin, 1962

timorensis (Miyagi, Toma \& Lien, 2004)

tonsus Edwards, 1924

Subgenus Gilesius Reinert, Harbach \& Kitching, 2006

alius Lien, 1968

pulchriventer (Giles, 1901)

Subgenus Gymnometopa Coquillett, 1905

mediovittatus (Coquillett, 1906)

Subgenus Halaedes Belkin, 1962

ashworthi Edwards, 1921

australis (Erichson, 1842)

wardangensis Brust, Ballard, Driver, Hartley, Galway \& Curran, 1998

Subgenus Himalaius Reinert, Harbach \& Kitching, 2006

gilli (Barraud, 1924)

simlensis Edwards, 1922

Subgenus Hopkinsius Reinert, Harbach \& Kitching, 2008

albocinctus (Barraud, 1924)

barnardi Edwards, 1924

embuensis Edwards, 1930

ingrami Edwards, 1930

nyasae Edwards, 1930

seoulensis Yamada, 1921

wellmanii (Theobald, 1905)

Subgenus Howardina Theobald, 1903

albonotatus (Coquillett, 1906)

allotecnon Kumm, Komp \& Ruiz, 1940

arborealis Bonne-Wepster \& Bonne, 1920

argyrites Dyar \& Núñez Tovar, 1927

aureolineatus Berlin, 1969

aurites (Theobald, 1907)

aurivittatus Cerqueira, 1943 
bahamensis Berlin, 1969

brevis Berlin, 1969

brevivittatus Berlin, 1969

busckii (Coquillett, 1906)

cozumelensis Díaz Nájera, 1966

ecuadoriensis Berlin, 1969

eleanorae Berlin, 1969

fulvithorax (Lutz, 1904)

grabhami Berlin, 1969

guatemala Berlin, 1969

guerrero Berlin, 1969

inaequalis (Grabham, 1907)

ioliota Dyar \& Knab, 1913

leei Berlin, 1969

lorraineae Berlin, 1969

marinkellei Berlin, 1969

martinezi Berlin, 1969

osornoi Berlin, 1969

pseudodominicii Komp, 1936

quadrivittatus (Coquillett, 1902)

septemstriatus Dyar \& Knab, 1907

sexlineatus (Theobald, 1901)

spinosus Berlin, 1969

stenei Thompson, 1956

vanemdeni Martini, 1931

walkeri (Theobald, 1901)

whitmorei Dunn, 1918

Subgenus Huaedes Huang, 1968

medialis (Brug, 1932)

variepictus (King \& Hoogstraal, 1946)

wauensis Huang, 1968

Subgenus Hulecoeteomyia Theobald, 1904

chrysolineatus (Theobald, 1907)

formosensis Yamada, 1921

harveyi (Barraud, 1923)

japonicus (Theobald, 1901)

japonicus amamiensis Tanaka, Mizusawa \& Saugstad, 1979

japonicus shintienensis Tsai \& Lien, 1950

japonicus yaeyamensis Tanaka, Mizusawa \& Saugstad, 1979

jugraensis (Leicester, 1908) 
koreicus (Edwards, 1917)

nigrorhynchus Brug, 1931

pallirostris Edwards, 1922

reinerti Rattanarithikul \& Harrison, 1988

rizali (Banks, 1906)

saxicola Edwards, 1922

sherki Knight, 1948

yunnanensis (Gaschen, 1934)

Subgenus Indusius Edwards, 1934

pulverulentus Edwards, 1922

Subgenus Isoaedes Reinert, 1979

cavaticus Reinert, 1979

Subgenus Jarnellius Reinert, Harbach \& Kitching, 2006

deserticola Zavortink, 1969

laguna Arnell \& Nielsen, 1972

monticola Belkin \& McDonald, 1957

sierrensis (Ludlow, 1905)

varipalpus (Coquillett, 1902)

Subgenus Jihlienius Reinert, Harbach \& Kitching, 2006

chungi Lien, 1968

gonguoensis Gong \& Lu, 1986

unicinctus Edwards, 1922

Subgenus Kenknightia Reinert, 1990

dissimilierodes Dong, Zhou \& Dong, 2002

dissimilis (Leicester, 1908)

gaffigani Reinert, 1990

harbachi Reinert, 1990

karwari (Barraud, 1924)

lerozeboomi Reinert, 1990

leucomeres (Giles, 1904)

litwakae Reinert, 1990

luzonensis Rozeboom, 1946

paradissimilis Rozeboom, 1946

pecori Reinert, 1990 
wilkersoni Reinert, 1990

Subgenus Kompia Aitken, 1941

purpureipes Aitken, 1941

Subgenus Leptosomatomyia Theobald, 1905

aurimargo Edwards, 1922

Subgenus Levua Stone \& Bohart, 1944

geoskusea Amos, 1944

Subgenus Lewnielsenius Reinert, Harbach \& Kitching, 2006

muelleri Dyar, 1920

Subgenus Lorrainea Belkin, 1962

amesii (Ludlow, 1903)

celebicus Mattingly, 1959

dasyorrhus King \& Hoogstraal, 1946

fumidus Edwards, 1928

lamelliferus Bohart \& Ingram, 1946

Subgenus Luius Reinert, Harbach \& Kitching, 2008

fengi Edwards, 1935

Subgenus Macleaya Theobald, 1903

calabyi Marks, 1963

elchoensis Taylor, 1929

humeralis Edwards, 1922

littlechildi Taylor, 1933

macmillani Marks, 1964

moloiensis Taylor, 1929

spinosipes Edwards, 1922

stoneorum Marks, 1977

tremulus (Theobald, 1903)

tulliae (Taylor, 1929)

wattensis Taylor, 1929 
Subgenus Molpemyia Theobald, 1910

auridorsum Edwards, 1922

pecuniosus Edwards, 1922

purpureus (Theobald, 1910)

Subgenus Mucidus Theobald, 1901

alternans (Westwood, 1835)

aurantius (Theobald, 1907)

aurantius chrysogaster (Taylor, 1927)

ferinus Knight, 1947

grahamii (Theobald, 1909)

laniger (Wiedemann, 1820)

lucianus Muspratt, 1959

mucidus (Karsch, 1887)

nigerrimus (Theobald, 1913)

nigrescens (Edwards, 1929)

quadripunctis (Ludlow, 1910)

quasiferinus Mattingly, 1961

scatophagoides (Theobald, 1901)

sudanensis (Theobald, 1908)

tonkingi Gebert, 1948

Subgenus Neomelaniconion Newstead, 1907

albicosta (Edwards, 1913)

albiradius (le Goff, Boussès \& Brunhes, 2007)

albothorax (Theobald, 1907)

aurovenatus Worth, 1960

belleci (le Goff, Boussès \& Brunhes, 2007)

bequaerti Wolfs, 1947

bergerardi Pajot \& Geoffroy, 1971

bolense Edwards, 1936

carteri Edwards, 1936

circumluteolum (Theobald, 1908)

crassiforceps Edwards, 1927

ellinorae Edwards, 1941

flavimargo Edwards, 1941

fontenillei (le Goff, Boussès \& Brunhes, 2007)

fuscinervis (Edwards, 1914)

jamoti Hamon \& Rickenbach, 1954

lineatopennis (Ludlow, 1905)

lineatopennis aureum (Gutsevich, 1955) 
luridus McIntosh, 1971

luteolateralis (Theobald, 1901)

mcintoshi Huang, 1985

monotrichus Edwards, 1936

nigropterum (le Goff, Boussès \& Brunhes, 2007)

palpale Newstead, 1907

pogonurus Edwards, 1936

punctocostalis (Theobald, 1909)

sylvaticum (le Goff, Boussès \& Brunhes, 2007)

taeniarostris (Theobald, 1909)

unidentatum Mclntosh, 1971

Subgenus Nyctomyia Harbach, 2013

pholeocolus (Linton \& Harbach, 2013)

biunguiculatus (Harbach, 2014)

Subgenus Ochlerotatus Lynch Arribalzaga, 1891

aboriginis Dyar, 1917

abserratus (Felt \& Young, 1904)

aculeatus (Theobald, 1903)

aenigmaticus Cerqueira \& Costa, 1946

akkeshiensis Tanaka, 1998

albescens Edwards, 1921

albifasciatus (Macquart, 1838)

albineus Séguy, 1923

aloponotum Dyar, 1917

ambreensis Rodhain \& Boutonnier, 1983

andersoni Edwards, 1926

angustivittatus Dyar \& Knab, 1907

annulipes (Meigen, 1830)

antipodeus (Edwards, 1920)

atactavittatus Arnell, 1976

atlanticus Dyar \& Knab, 1906

auratus Grabham, 1906

aurifer (Coquillett, 1903)

bancroftianus Edwards, 1921

behningi Martini, 1926

bejaranoi Martinez, Carcavallo \& Prosen, 1960

berlandi Séguy, 1921

bicristatus Thurman \& Winkler, 1950

bimaculatus (Coquillett, 1902)

biskraensis Brunhes, 1999 
bogotanus (Arnell, 1976)

breedensis Muspratt, 1953

burjaticus (Kuchartshuk, 1973)

burpengaryensis (Theobald, 1905)

caballus (Theobald, 1912)

cacozelus Marks, 1963

calcariae Marks, 1957

calumnior Belkin, Heinemann \& Page, 1970

campestris Dyar \& Knab, 1907

camptorhynchus (Thomson, 1869)

canadensis (Theobald, 1901)

canadensis mathesoni Middlekauff

cantans (Meigen, 1818)

cantator (Coquillett, 1903)

caspius (Pallas, 1771)

caspius hargreavesi Edwards, 1920

caspius meirae Ribeiro, Ramos, Capela \& Pires, 1980

cataphylla Dyar, 1916

chelli (Edwards, 1915)

churchillensis Ellis \& Brust, 1973

clelandi (Taylor, 1914)

clivis Lanzaro \& Eldridge, 1992

coluzzii Rioux, Guilvard \& Pasteur, 1998

comitatus Arnell, 1976

communis (de Geer, 1776)

condolescens Dyar \& Knab, 1907

continentalis Dobrotworsky, 1960

crinifer (Theobald, 1903)

culiciformis (Theobald, 1905)

cunabulanus Edwards, 1924

cyprioides Danilov \& Stupin, 1982

cyprius Ludlow, 1920

dahlae (Nielsen, 2009)

decticus Howard, Dyar \& Knab, 1917

deficiens Arnell, 1976

detritus Haliday, 1833

diantaeus Howard, Dyar \& Knab, 1913

dorsalis (Meigen, 1830)

dufouri Hamon, 1953

duplex Martini, 1926

dupreei (Coquillett, 1904)

dzeta Séguy, 1924

edgari Stone \& Rosen, 1952

eidsvoldensis Mackerras, 1927 
eucephalaeus Dyar, 1918

euedes Howard, Dyar \& Knab, 1913

euiris Dyar, 1922

euplocamus Dyar \& Knab, 1906

excrucians (Walker, 1856)

explorator Marks, 1964

fitchii (Felt \& Young, 1904)

flavescens (Müller, 1764)

flavifrons (Skuse, 1889)

fulvus (Wiedemann, 1828)

fulvus pallens Ross, 1943

grossbecki Dyar \& Knab, 1906

gutzevichi Dubitsky \& Deshevykh, 1978

hakusanensis Yamaguti \& Tamaboko, 1954

harrisoni Muspratt, 1953

hastatus Dyar, 1922

hesperonotius Marks, 1959

hexodontus Dyar, 1916

hodgkini Marks, 1959

hokkaidensis Tanaka, Mizusawa \& Saugstad, 1979

hungaricus Mihalyi, 1955

imperfectus Dobrotworsky, 1962

impiger (Walker, 1848)

impiger daisetsuzanus Tanaka, Mizusawa \& Saugstad, 1979

implicatus Vockeroth, 1954

incomptus Arnell, 1976

increpitus Dyar, 1916

inexpectatus Bonne-Wepster, 1948

infirmatus Dyar \& Knab, 1906

intermedius Danilov \& Gornostaeva, 1987

intrudens Dyar, 1919

jacobinae Serafim \& Davis, 1933

jorgi Carpintero \& Leguizamón, 2000

juppi McIntosh, 1973

kasachstanicus Gutsevich, 1962

krymmontanus Alekseev, 1989

lasaensis Meng, 1962

lasaensis gyirongensis Ma, 1982

lepidonotus Edwards, 1920

lepidus Cerqueira \& Paraense, 1945

leucomelas (Meigen, 1804)

linesi Marks, 1964

longifilamentus Su \& Zhang, 1988

luteifemur Edwards, 1926 
macintoshi Marks, 1959

martineti Senevet, 1937

mcdonaldi Belkin, 1962

melanimon Dyar, 1924

meprai Martinez \& Prosen, 1953

mercurator Dyar, 1920

milleri Dyar, 1922

mitchellae (Dyar, 1905)

montchadskyi Dubitsky, 1968

multiplex (Theobald, 1903)

nevadensis Chapman \& Barr, 1964

nigrinus (Eckstein, 1918)

nigripes (Zetterstedt, 1838)

nigrithorax (Macquart, 1847)

nigrocanus Martini, 1927

nigromaculis (Ludlow, 1906)

niphadopsis Dyar \& Knab, 1918

nivalis Edwards, 1926

normanensis (Taylor, 1915)

nubilus Theobald, 1903

obturbator Dyar \& Knab, 1907

oligopistus Dyar, 1918

patersoni Shannon \& del Ponte, 1928

pectinatus Arnell, 1976

pennai Antunes \& Lane, 1938

perkinsi Marks, 1949

pertinax Grabham, 1906

phaecasiatus Marks, 1964

phaeonotus Arnell, 1976

pionips Dyar, 1919

postspiraculosus Dobrotworsky, 1961

procax (Skuse, 1889)

provocans (Walker, 1848)

pseudonormanensis Marks, 1949

pulcritarsis (Rondani, 1872)

pulcritarsis asiaticus Edwards, 1926

pullatus (Coquillett, 1904)

punctodes Dyar, 1922

punctor (Kirby, 1837)

purpuraceus Brug, 1932

purpureifemur Marks, 1959

purpuriventris Edwards, 1926

quasirusticus Torres Cañamares, 1951

ratcliffei Marks, 1959 
raymondi del Ponte, Castro \& García, 1951

refiki Medschid, 1928

rempeli Vockeroth, 1954

rhyacophilus da Costa Lima, 1933

riparioides Su \& Zhang, 1987

riparius Dyar \& Knab, 1907

rusticus (Rossi, 1790)

rusticus subtrichurus Martini, 1927

rusticus trichurus (Dyar, 1904)

sagax (Skuse, 1889)

sapiens Marks, 1964

scapularis (Rondani, 1848)

schizopinax Dyar, 1929

schtakelbergi Shingarev, 1928

scutellalbum Boshell-Manrique, 1939

sedaensis Lei, 1989

sergievi Danilov, Markovich \& Proskuryakova, 1978

serratus (Theobald, 1901)

shannoni Vargas \& Downs, 1950

silvestris (Dobrotworsky, 1961)

simanini Gutsevich, 1966

sinkiangensis Hsiao, 1977

sollicitans (Walker, 1856)

spencerii (Theobald, 1901)

spencerii idahoensis (Theobald, 1903)

spilotus Marks, 1963

squamiger (Coquillett, 1902)

sticticus (Meigen, 1838)

stigmaticus Edwards, 1922

stimulans (Walker, 1848)

stramineus Dubitzky, 1970

stricklandi (Edwards, 1912)

subalbirostris Klein \& Marks, 1960

subdiversus Martini, 1926

surcoufi (Theobald, 1912)

synchytus Arnell, 1976

taeniorhynchus (Wiedemann, 1821)

tahoensis Dyar, 1916

thelcter Dyar, 1918

theobaldi (Taylor, 1914)

thibaulti Dyar \& Knab, 1910

tormentor Dyar \& Knab, 1906

tortilis (Theobald, 1903)

trivittatus (Coquillett, 1902) 
turneri Marks, 1963

upatensis Anduze \& Hecht, 1943

ventrovittis Dyar, 1916

vigilax (Skuse, 1889)

vigilax ludlowae (R. Blanchard)

vigilax vansomerenae Mattingly

vittiger (Skuse, 1889)

washinoi Lanzaro \& Eldridge, 1992

Subgenus Paraedes Edwards, 1934

barraudi (Edwards, 1934)

bonneae Mattingly, 1958

chrysoscuta (Theobald, 1910)

collessi Mattingly, 1958

menoni Mattingly, 1958

ostentatio (Leicester, 1908)

pagei (Ludlow, 1911)

thailandensis Reinert, 1976

Subgenus Patmarksia Reinert, Harbach \& Kitching, 2006

anggiensis Bonne-Wepster, 1937

argenteitarsis Brug, 1932

argyronotum Belkin, 1962

buxtoni Belkin, 1962

clintoni Taylor, 1946

derooki Brug, 1932

dobodurus King \& Hoogstraal, 1946

hollandius King \& Hoogstraal, 1946

mackerrasi Taylor, 1927

novalbitarsis King \& Hoogstraal, 1946

palmarum Edwards, 1924

papuensis (Taylor, 1914)

subalbitarsis King \& Hoogstraal, 1946

Subgenus Petermattinglyius Reinert, Harbach \& Kitching, 2009

franciscoi Mattingly, 1959

iyengari Edwards, 1923

punctipes Edwards, 1921

scanloni Reinert, 1970

whartoni Mattingly, 1965 
Subgenus Phagomyia Theobald, 1905

assamensis (Theobald, 1908)

cacharanus (Barraud, 1923)

cogilli Edwards, 1922

deccanus (Barraud, 1923)

feegradei Barraud, 1934

gubernatoris (Giles, 1901)

gubernatoris kotiensis Barraud, 1934

inquinatus Edwards, 1922

iwi Marks, 1955

khazani Edwards, 1922

kiangsiensis Tung, 1955

lophoventralis (Theobald, 1910)

melanopterus (Giles, 1904)

plumiferus King \& Hoogstraal, 1946

prominens (Barraud, 1923)

stevensoni (Barraud, 1923)

watasei Yamada, 1921

Subgenus Polyleptiomyia Theobald, 1905

albocephalus (Theobald, 1903)

gandarai da Cunha Ramos, Capela \& Ribeiro, 1995

Subgenus Pseudoarmigeres Stone \& Knight, 1956

albomarginatus (Newstead, 1907)

argenteoventralis (Theobald, 1909)

argenteoventralis dunni (Evans, 1928)

kummi Edwards, 1930

michaelikati van Someren, 1946

michaelikati gurneri van Someren, 1946

natalensis Edwards, 1930

Subgenus Rampamyia Reinert, Harbach \& Kitching, 2006

albilabris Edwards, 1925

notoscriptus (Skuse, 1889)

notoscriptus montanus Brug, 1939

quinquelineatus Edwards, 1922

Subgenus Rhinoskusea Edwards, 1929 
longirostris (Leicester, 1908)

pillaii Mattingly, 1958

portonovoensis Tewari \& Hiriyan, 1992

wardi Reinert, 1976

Subgenus Sallumia Reinert, Harbach \& Kitching, 2008

hortator Dyar \& Knab, 1907

perventor Cerqueira \& Costa, 1946

Subgenus Scutomyia Theobald, 1904

albolineatus (Theobald, 1904)

arboricola Knight \& Rozeboom, 1946

bambusicola Knight \& Rozeboom, 1946

boharti Knight \& Rozeboom, 1946

hoogstraali Knight \& Rozeboom, 1946

impatibilis (Walker, 1860)

laffooni Knight \& Rozeboom, 1946

platylepidus Knight \& Hull, 1951

pseudalbolineatus Brug, 1939

Subgenus Skusea Theobald, 1903

cartroni (Ventrillon, 1906)

lambrechti van Someren, 1971

moucheti Ravaonjanahary \& Brunhes, 1977

pembaensis Theobald, 1901

Subgenus Stegomyia Theobald, 1901

aegypti (Linnaeus, 1762)

aegypti formosus (Walker, 1848)

africanus (Theobald, 1901)

agrihanensis Bohart, 1957

albopictus (Skuse, 1895)

alcasidi Huang, 1972

alorensis Bonne-Wepster \& Brug, 1932

amaltheus de Meillon \& Lavoipierre, 1944

andrewsi Edwards, 1926

angustus Edwards, 1935

annandalei (Theobald, 1910)

aobae Belkin, 1962

apicoargenteus (Theobald, 1909) 
bambusae Edwards, 1935

blacklocki Evans, 1925

bromeliae (Theobald, 1911)

burnsi Basio \& Reisen, 1971

calceatus Edwards, 1924

chaussieri Edwards, 1923

chemulpoensis Yamada, 1921

contiguus Edwards, 1936

cooki Belkin, 1962

corneti Huang, 1986

craggi (Barraud, 1923)

cretinus Edwards, 1921

daitensis Miyagi \& Toma, 1981

deboeri Edwards, 1926

demeilloni Edwards, 1936

denderensis Wolfs, 1949

dendrophilus Edwards, 1921

desmotes (Giles, 1904)

dybasi Bohart, 1957

ealaensis Huang, 2004

edwardsi (Barraud, 1923)

ethiopiensis Huang, 2004

flavopictus Yamada, 1921

flavopictus downsi Bohart \& Ingram, 1946

flavopictus miyarai Tanaka, Mizusawa \& Saugstad, 1979

fraseri (Edwards, 1912)

futunae Belkin, 1962

galloisi Yamada, 1921

galloisiodes Liu \& Lu, 1984

gandaensis Huang, 2004

gardnerii (Ludlow, 1905)

gardnerii imitator (Leicester, 1908)

grantii (Theobald, 1901)

guamensis Farner \& Bohart, 1944

gurneyi Stone \& Bohart, 1944

hakanssoni Knight \& Hurlbut, 1949

hansfordi Huang, 1997

hebrideus Edwards, 1926

heischi van Someren, 1951

hensilli Farner, 1945

hogsbackensis Huang, 2004

hoguei Belkin, 1962

horrescens Edwards, 1935

josiahae Huang, 1988 
katherinensis Woodhill, 1949

keniensis van Someren, 1946

kenyae van Someren, 1946

kesseli Huang \& Hitchcock, 1980

kivuensis Edwards, 1941

krombeini Huang, 1975

langata van Someren, 1946

ledgeri Huang, 1981

lilii (Theobald, 1910)

luteocephalus (Newstead, 1907)

maehleri Bohart, 1957

malayensis Colless, 1962

malikuli Huang, 1973

marshallensis Stone \& Bohart, 1944

mascarensis MacGregor, 1924

masseyi Edwards, 1923

mattinglyorum Huang, 1994

maxgermaini Huang, 1990

mediopunctatus (Theobald, 1905)

mediopunctatus sureilensis Barraud, 1934

metallicus (Edwards, 1912)

mickevichae Huang, 1988

mpusiensis Huang, 2004

muroafcete Huang, 1997

neoafricanus Cornet, Valade \& Dieng, 1978

neogalloisi Chen \& Chen, 2000

neopandani Bohart, 1957

njombiensis Huang, 1997

novalbopictus Barraud, 1931

opok Corbet \& van Someren, 1962

palauensis Bohart, 1957

pandani Stone, 1939

patriciae Mattingly, 1954

paullusi Stone \& Farner, 1945

pernotatus Farner \& Bohart, 1944

perplexus (Leicester, 1908)

polynesiensis Marks, 1951

poweri (Theobald, 1905)

pseudalbopictus (Borel, 1928)

pseudoafricanus Chwatt, 1949

pseudonigeria (Theobald, 1910)

pseudoscutellaris (Theobald, 1910)

quasiscutellaris Farner \& Bohart, 1944

rhungkiangensis Chang \& Chang, 1974 
riversi Bohart \& Ingram, 1946

robinsoni Belkin, 1962

rotanus Bohart \& Ingram, 1946

rotumae Belkin, 1962

ruwenzori Haddow \& van Someren, 1950

saimedres Huang, 1988

saipanensis Stone, 1945

sampi Huang, 2004

schwetzi Edwards, 1926

scutellaris (Walker, 1858)

scutoscriptus Bohart \& Ingram, 1946

seampi Huang, 1974

seatoi Huang, 1969

segermanae Huang, 1997

sibiricus Danilov \& Filippova, 1978

simpsoni (Theobald, 1905)

soleatus Edwards, 1924

strelitziae Muspratt, 1950

subalbopictus Barraud, 1931

subargenteus Edwards, 1925

tabu Ramalingam \& Belkin, 1965

tongae Edwards, 1926

tulagiensis Edwards, 1926

unilineatus (Theobald, 1906)

upolensis Marks, 1957

usambara Mattingly, 1953

varuae Belkin, 1962

vinsoni Mattingly, 1953

$w$-albus (Theobald, 1905)

wadai Tanaka, Mizusawa \& Saugstad, 1979

woodi Edwards, 1922

Subgenus Tanakaius Reinert, Harbach \& Kitching, 2004

savoryi Bohart, 1957

togoi (Theobald, 1907)

Subgenus Tewarius Reinert, 2006

agastyai Tewari \& Hiriyan, 1992

nummatus Edwards, 1923

pseudonummatus Reinert, 1973

reubenae Tewari \& Hiriyan, 1992 
Subgenus Vansomerenis Reinert, Harbach \& Kitching, 2006

luteostriatus Robinson, 1950

pulchrithorax Edwards, 1939

hancocki van Someren, 1962

Subgenus Zavortinkius Reinert, 1999

brunhesi Reinert, 1999

brygooi Brunhes, 1971

fulgens (Edwards, 1917)

geoffroyi Reinert, 1999

huangae Reinert, 1999

interruptus Reinert, 1999

longipalpis (Grünberg, 1905)

monetus Edwards, 1935

mzooi van Someren, 1962

phillipi van Someren, 1949

pollinctor (Graham, 1910)

Subgenus uncertain

aitkeni Schick, 1970

alboapicus Schick, 1970

alticolaa Bonne-Wepster, 1948

amabilis Schick, 1970

argyrothorax Bonne-Wepster \& Bonne, 1920

auronitens Edwards, 1922

australiensis (Theobald, 1910)

berlini Schick, 1970

bertrami Schick, 1970

biocellatus (Taylor, 1915)

braziliensis Gordon \& Evans, 1922

brelandi Zavortink, 1972

britteni Marks \& Hodgkin, 1958

buenaventura Schick, 1970

burgeri Zavortink, 1972

campana Schick, 1970

candidoscutellum Marks, 1947

casali Schick, 1970

chionotum Zavortink, 1972

crossi Lien, 1967

daliensis (Taylor, 1916)

daryi Schick, 1970 
diazi Schick, 1970

eatoni (Edwards, 1916)

gabriel Schick, 1970

galindoi Schick, 1970

gracilelineatus Bonne-Wepster, 1937

hendersoni Cockerell, 1918

heteropus Dyar, 1921

homoeopus Dyar, 1922

idanus Schick, 1970

impostor Schick, 1970

insolitus (Coquillett, 1906)

keefei King \& Hoogstraal, 1946

knabi (Coquillett, 1906)

kompi Vargas \& Downs, 1950

koreicoides Sasa, Kano \& Hayashi, 1950

lauriei (Carter, 1920)

mallochi Taylor, 1944

metoecopus Dyar, 1925

monocellatus Marks, 1948

niveoscutum Zavortink, 1972

oreophilus (Edwards, 1916)

peipingensis Feng, 1938

plagosus Marks, 1959

podographicus Dyar \& Knab, 1906

quasirubithorax (Theobald, 1910)

roai Belkin, 1962

rubiginosus Belkin, 1962

sandrae Zavortink, 1972

schicki Zavortink, 1972

schroederi Schick, 1970

sintoni (Barraud, 1924)

stanleyi Peters, 1963

subauridorsum Marks, 1948

suffusus Edwards, 1922

sumidero Schick, 1970

tehuantepec Schick, 1970

terrens (Walker, 1856)

thorntoni Dyar \& Knab, 1907

toxopeusi Bonne-Wepster, 1948

triseriatus (Say, 1823)

tsiliensis King \& Hoogstraal, 1946

vargasi Schick, 1970

versicolor (Barraud, 1924)

wasselli Marks, 1947 
zavortinki Schick, 1970

zoosophus Dyar \& Knab, 1918

Not placed by Sathe \& Girhe, 2002

kolhapuriensis Sathe \& Girhe, 2002

panchgangee Sathe \& Girhe, 2002

sangitee Sathe \& Girhe, 2002

sangiti Girhe \& Sathe, 2001

\section{Genus Armigeres}

Armigeres Theobald, 1901

alkatirii Toma, Miyagi \& Syafruddin, 1995

apoensis Bohart \& Farner, 1944

aureolineatus (Leicester, 1908)

azurini Basio, 1971

baisasi Stone \& Thurman, 1958

bhayungi Thurman \& Thurman, 1958

breinli (Taylor, 1914)

candelabrifer Brug, 1939

confusus Edwards, 1915

conjungens Edwards, 1914

denbesteni Brug, 1925

durhami (Edwards, 1917)

ejercitoi Baisas, 1935

fimbriatus Edwards, 1930

foliatus Brug, 1931

giveni Edwards, 1926

hybridus Edwards, 1914

joloensis (Ludlow, 1904)

jugraensis (Leicester, 1908)

kesseli Ramalingam, 1987

kinabaluensis Ramalingam, 1972

kuchingensis Edwards, 1915

lacuum Edwards, 1922

laoensis Toma \& Miyagi, 2003

maiae (Edwards, 1917)

malayi (Theobald, 1901)

manalangi Baisas, 1935

maximus Edwards, 1922

milnensis Lee, 1944

moultoni Edwards, 1914 
obturbans (Walker, 1859)

pallithorax Dong, Zhou \& Dong, 2004

papuensis Peters, 1963

setifer Delfinado, 1966

seticoxitus Luh \& Li, 1981

subalbatus (Coquillett, 1898)

subalbatus chrysocorporis Hsieh \& Liao, 1956

theobaldi Barraud, 1934

yunnanensis Dong, Zhou \& Dong, 1995

Leicesteria Theobald, 1904

annulipalpis (Theobald, 1910)

annulitarsis (Leicester, 1908)

balteatus Macdonald, 1960

cingulatus (Leicester, 1908)

dentatus Barraud, 1927

digitatus (Edwards, 1914)

dolichocephalus (Leicester, 1908)

flavus (Leicester, 1908)

inchoatus Barraud, 1927

lepidocoxitus Dong, Zhou \& Dong, 1995

longipalpis (Leicester, 1904)

magnus (Theobald, 1908)

menglaensis Dong, Zhou \& Dong, 2002

omissus (Edwards, 1914)

pectinatus (Edwards, 1914)

pendulus (Edwards, 1914)

traubi Macdonald, 1960

vimoli Thurman \& Thurman, 1958

\section{Genus Eretmapodites}

adami Ferrara \& Eouzan, 1974

angolensis da Cunha Ramos \& Ribeiro, 1992

argyrurus Edwards, 1936

brenguesi Rickenbach \& Lombrici, 1975

brottesi Rickenbach, 1967

caillardi Rickenbach, Ferrara \& Eouzan, 1968

chrysogaster Graham, 1909

corbeti Hamon, 1962

dracaenae Edwards, 1916

dundo da Cunha Ramos \& Ribeiro, 1992

eouzani Rickenbach \& Lombrici, 1974 
ferrarai Rickenbach \& Eouzan, 1970

forcipulatus Edwards, 1936

germaini Rickenbach \& Eouzan, 1970

gilletti van Someren, 1949

grahami Edwards, 1911

grenieri Hamon \& van Someren, 1961

haddowi van Someren, 1949

hamoni Grjebine, 1972

harperi van Someren, 1949

hightoni van Someren, 1947

inornatus Newstead, 1907

intermedius Edwards, 1936

jani Rickenbach \& Lombrici, 1976

lacani Rickenbach \& Eouzan, 1970

leucopous Graham, 1909

mahaffyi van Someren, 1949

marcellei Adam \& Hamon, 1959

mattinglyi Hamon \& van Someren, 1961

melanopous Graham, 1909

mortiauxi Cunha Ramos \& Ribeiro, 1990

oedipodeios Graham, 1909

parvipluma Edwards, 1941

pauliani Grjebine, 1950

penicillatus Edwards, 1941

plioleucus Edwards, 1941

plioleucus brevis Edwards, 1941

productus Edwards, 1941

quinquevittatus Theobald, 1901†

ravissei Rickenbach \& Eouzan, 1970

rickenbachi Ferrara \& Eouzan, 1974

salauni Rickenbach, Ferrara \& Eouzan, 1968

semisimplicipes Edwards, 1914

silvestris Ingram \& de Meillon, 1927

silvestris conchobius Edwards, 1941

subsimplicipes Edwards, 1914

tendeiroi da Cunha Ramos, Ribeiro \& Machado, 1992

tonsus Edwards, 1941

vansomereni Hamon, 1962

wansoni Edwards, 1941

wansoni douceti Adam \& Hamon, 1959

\section{Genus Haemagogus}

Subgenus Conopostegus Dyar, 1925 
clarki (Galindo, Carpenter \& Trapido, 1953)

leucocelaenus (Dyar \& Shannon, 1924)

leucophoebus (Galindo, Carpenter \& Trapido, 1953)

leucotaeniatus (Komp, 1938)

Subgenus Haemagogus Williston, 1896

acutisentis Arnell, 1973

aeritinctus Galindo \& Trapido, 1967

albomaculatus Theobald, 1903

anastasionis Dyar, 1921

andinus Osorno-Mesa, 1944

argyromeris Dyar \& Ludlow, 1921

baresi Cerqueira, 1960

boshelli Osorno-Mesa, 1944

capricornii Lutz, 1904

celeste Dyar \& Nuñez Tovar, 1927

chalcospilans Dyar, 1921

chrysochlorus Arnell, 1973

equinus Theobald, 1903

iridicolor Dyar, 1921

janthinomys Dyar, 1921

lucifer (Howard, Dyar \& Knab, 1913)

mesodentatus Komp \& Kumm, 1938

nebulosus Arnell, 1973

panarchys Dyar, 1921

regalis Dyar \& Knab, 1906

soperi Levi-Castillo, 1955

spegazzinii Brethès, 1912

splendens Williston, 1896

tropicalis Cerqueira \& Antunes, 1938

\section{Genus Heizmannia}

Subgenus Heizmannia Ludlow, 1905

aurea Brug, 1932

aureochaeta (Leicester, 1908)

carteri Amerasinghe, 1993

chandi Edwards, 1922

chengi Lien, 1968

communis (Leicester, 1908)

complex (Theobald, 1910) 
covelli Barraud, 1929

demeilloni Mattingly, 1970

funerea (Leicester, 1908)

greenii (Theobald, 1905)

heterospina Gong \& Lu, 1986

himalayensis Edwards, 1922

indica (Theobald, 1905)

kana Tanaka, Mizusawa \& Saugstad, 1979

kanhsienensis Tung, 1955

lii Wu, 1936

macdonaldi Mattingly, 1957

mattinglyi Thurman, 1959

menglianensis Lu \& Gong, 1986

menglianeroides Dong, Dong \& Zhou, 2003

persimilis Mattingly, 1970

propinqua Mattingly, 1970

proxima Mattingly, 1970

reidi Mattingly, 1957

ruiliensis Dong, Zhou \& Wang, 1997

scanloni Mattingly, 1970

scintillans Ludlow, 1905

taiwanensis Lien, 1968

tengchongensis Dong, Wang \& Zhou, 2002

thelmae Mattingly, 1970

viridis Barraud, 1929

Subgenus Mattinglyia Lien, 1968

achaetae (Leicester, 1908)

catesi (Lien, 1968)

discrepans (Edwards, 1922)

lui Gong \& Li, 1999

occidentayunnana (Gong \& Lu, 1991)

tripunctata (Theobald, 1908)

\section{Genus Opifex}

Subgenus Nothoskusea Dumbleton, 1962

chathamicus (Dubleton, 1962)

Subgenus Opifex Hutton, 1902

fuscus Hutton, 1902

\section{Genus Psorophora}


Subgenus Grabhamia Theobald, 1903

cingulata (Fabricius, 1805)

columbiae (Dyar \& Knab, 1906)

confinnis (Lynch Arribálzaga, 1891)

dimidiata Cerqueira, 1943

discolor (Coquillett, 1903)

infinis (Dyar \& Knab, 1906)

insularia (Dyar \& Knab, 1906)

jamaicensis Theobald, 1901

leucocnemis Martini, 1931

paulli Paterson \& Shannon, 1927

pruinosa Martini, 1935

pygmaea (Theobald, 1903)

santamarinai Broche, 2000

signipennis (Coquillett, 1904)

varinervis Edwards, 1922

Subgenus Janthinosoma Lynch Arribálzaga, 1891

albigenu (Peryassú, 1908)

albipes (Theobald, 1907)

amazonica Cerqueira, 1960

champerico (Dyar \& Knab, 1906)

circumflava Cerqueira, 1943

cyanescens (Coquillett, 1902)

discrucians (Walker, 1856)

ferox (von Humboldt, 1819)

fiebrigi Edwards, 1922

forceps Cerqueira, 1939

horrida (Dyar \& Knab, 1908)

johnstonii (Grabham, 1905)

lanei Shannon \& Cerqueira, 1943

longipalpus Randolph \& O'Neill, 1944

lutzii (Theobald, 1901)

mathesoni Belkin \& Heinemann, 1975

melanota Cerqueira, 1943

mexicana (Bellardi, 1859)

pilosa Duret, 1971

pseudoalbipes Duret, 1971

pseudomelanota Barata \& Cotrim, 1971

totonaci Lassmann, 1951

varipes (Coquillett, 1904) 
Subgenus Psorophora Robineau-Desvoidy, 1827

ciliata (Fabricius, 1794)

cilipes (Fabricius, 1805)

holmbergii Lynch Arribálzaga, 1891

howardii Coquillett, 1901

lineata (von Humboldt, 1819)

ochripes (Macquart, 1850)

pallescens Edwards, 1922

pilipes (Macquart, 1834)

saeva Dyar \& Knab, 1906

stonei Vargas, 1956

Nomina dubia

goeldii (Giles, 1904)

marmorata (Philippi, 1865)

perterrens (Walker, 1856)

Genus Udaya Thurman, 1954

argyrurus (Edwards, 1934)

lucaris Macdonald \& Mattingly, 1960

subsimilis (Barraud, 1927)

\section{Genus Verrallina}

Subgenus Harbachius Reinert, 1999

abdita (Barraud, 1931)

consonensis (Reinert, 1973)

fragilis Leicester, 1908

hamistylus (Laffoon, 1946)

indecorabilis Leicester, 1908

nobukonis (Yamada, 1932)

pahangi (Delfinado, 1968)

ramalingami (Reinert, 1974)

robertsi (Laffoon, 1946)

srilankensis (Reinert, 1977)

stunga (Klein, 1973)

uniformis (Theobald, 1910)

yusafi (Barraud, 1931)

Subgenus Neomacleaya Theobald, 1907 


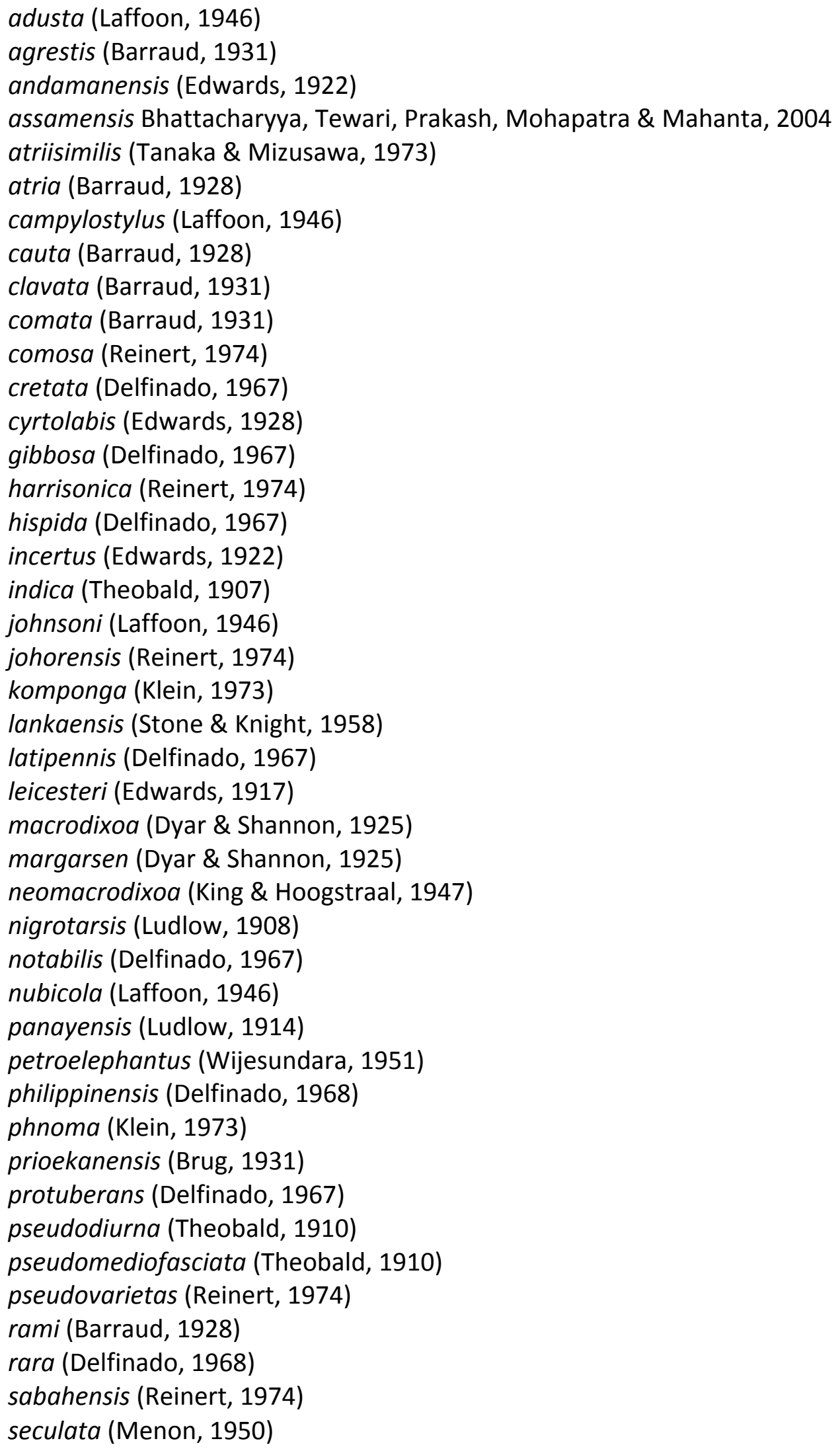


singularis (Leicester, 1908)

sohni (Reinert, 1974)

spermathecus (Wijesundara, 1951)

torosa (Delfinado, 1967)

uncus (Theobald, 190l)

vallistris (Barraud, 1928)

varietas (Leicester, 1908)

virilis Leicester, 1908

yerburyi (Edwards, 1917)

Subgenus Verrallina Theobald, 1903

azureosquamata (Bonne-Wepster, 1948)

bifoliata (King \& Hoogstraal, 1947)

butleri (Theobald, 1901)

carmenti (Edwards, 1924)

cuccioi (Belkin, 1962)

cunninghami (Taylor, 1944)

dux (Dyar \& Shannon, 1925)

embiensis (Huang, 1968)

foliformis (King \& Hoogstraal, 1947)

funerea (Theobald, 1903)

iriomotensis (Tanaka \& Mizusawa, 1973)

killertonis (Huang, 1968)

leilae (King \& Hoogstraal, 1947)

lineata (Taylor, 1914)

lugubris (Barraud, 1928)

mccormicki (Belkin, 1962)

milnensis (King \& Hoogstraal, 1947)

multifolium (King \& Hoogstraal, 1947)

obsoleta (Huang, 1968)

parasimilis (King \& Hoogstraal, 1947)

pipkini (Bohart, 1957)

quadrifolium (Brug, 1934)

quadrispinata (King \& Hoogstraal, 1947)

reesi (King \& Hoogstraal, 1947)

sentania (King \& Hoogstraal, 1947)

similis (Theobald, 1910)

simpla (King \& Hoogstraal, 1947)

trispinata (King \& Hoogstraal, 1947)

vanapa (Huang, 1968)

variabilis (Huang, 1968)

Nomen dubium 
perdita Leicester, 1908

Genus Zeugnomyia Leicester, 1908

aguilari Baisas \& Feliciano, 1953

fajardoi Baisas \& Feliciano, 1953

gracilis Leicester, 1908

lawtoni Baisas, 1946 